\title{
EMPRUNTS LEXICAUX À L'ALLEMAND DANS LE FRANÇAIS
}

\author{
Zuzana Navrátilová
}

\begin{abstract}
The paper deals with German loan-words in French; its aim is to study relative frequency in the written language and to show to what degree they are known and used by contemporary native speakers. The base of the research is the monolingual and etymologic dictionary Trésor de la langue française, from which a corpus of lexemes determinated to further analysis was isolated. It was quite problematic to determine the criteria for drawing up the corpus and to define the term German loan-word. The frequency of the chosen lexemes is hereafter studied in publicistic texts. The final stage of the work is focused on a research on knowledge and usage (by contemporary French native speakers) of those lexical units, which are used very rarely in the written language.
\end{abstract}

Keywords: German loan-word; relative frequency; Trésor de la langue française; etymology; classification of loan-words.

Résumé : L'article est consacré aux emprunts lexicaux à l'allemand dans le français. Plus concrètement, l'auteur se concentre sur la fréquence relative de ceux-ci dans la langue écrite et aussi à la mesure de leur connaissance et de leur utilisation par les natifs actuels. Le point de départ de la recherche est le dictionnaire historique et étymologique Trésor de la langue française à partir duquel a été isolé, par une sélection détaillée, un ensemble de lexèmes destinés à l'analyse. Le choix même des critères pour la délimitation de ce corpus de travail, aussi bien que la définition du terme emprunt lexical à l'allemand s'est révélée être assez problématique. La fréquence des lexèmes choisis est ensuite étudiée grâce aux articles de presse. La phase finale du travail consistera en une recherche sur la connaissance et l'utilisation de telles unités lexicales, dont la fréquence relative en langue écrite est faible, chez les locuteurs français actuels.

Mots clés : emprunt lexical à l'allemand ; fréquence relative ; Trésor de la langue française ; étymologie ; classification des emprunts.

\section{Sujet et objectifs}

Dans mon mémoire de thèse, je fais le lien avec le programme de recherche TLF-Étym ${ }^{1}$ auquel j'ai eu l'honneur de participer en 2008 au CNRS/ AtILF (Université Nancy 2). Le projet se fixe pour objectif la mise à jour des notices étymologiques du Trésor de la langue française (informatisé) $)^{2}$, et lors de ma participation à ce projet, je me suis consacrée à une analyse étymologique détaillée de quatorze emprunts lexicaux à l'allemand qui, parmi de nombreux autres, figurent dans le TLF(i).

\footnotetext{
www.atilf.fr/tlf-etym.

$2 T L F(i)$.
} 
Pendant mes études doctorales, j’ai décidé de m’orienter vers les emprunts lexicaux à l'allemand dans un cadre plus large et le but de ma thèse est concrètement la recherche de la fréquence de ceux-ci dans la langue écrite de nos jours, ainsi que la mesure de leur connaissance et de leur utilisation par les locuteurs natifs actuels.

Le point de départ pour la délimitation de mon corpus de travail était une fois de plus le $T L F(i)$ à partir duquel j'ai isolé, par une sélection détaillée, un ensemble de 105 lexèmes d'origine allemande. La fréquence des lexèmes choisis a été ensuite étudiée sur le matériel des articles de presse, précisément à l'aide des versions informatisées de quatre journaux et périodiques français principaux ; Le Monde, Libération, Le Figaro et Le Nouvel Observateur. Au niveau temporel, $\mathrm{j}^{\prime}$ ai limité la recherche aux textes publiés après le $1^{\text {er }}$ janvier 2000. La phase finale de mon travail consistera en une recherche sur la connaissance et l'utilisation de telles unités lexicales dont la fréquence relative en langue écrite est faible, chez les locuteurs français actuels. Cette dernière étape sera effectuée sous forme d'un questionnaire. ${ }^{3}$

\section{Emprunts lexicaux à l'allemand dans le français - critères de délimitati- on du corpus de travail et classification}

Dans ma contribution, j'aimerais me consacrer à la définition du terme emprunt lexical à l'allemand, au choix des critères pour la création du corpus de travail initial et à la classification des unités lexicales ainsi définies car ces quelques étapes de base se sont déjà révélées être assez problématiques.

\subsection{Définition de la notion emprunt lexical à l'allemand}

Pour désigner les unités lexicales qui ont été empruntées à l'allemand, on utilise souvent le terme germanisme. Celui-ci est défini par le TLF(i) comme " tournure propre à une langue germanique, plus particulièrement à l'allemand, ne pouvant être traduite littéralement dans une autre langue », ou bien comme "terme emprunté à une langue germanique ". Je considère donc que si l'on envisage une précision terminologique, il est à recommander d'utiliser plutôt la notion d'emprunt à l'allemand ; on évitera ainsi la confusion avec des lexèmes peut-être bien d'origine germanique mais différente de l'allemand.

\subsection{Le nombre des emprunts lexicaux à l'allemand dans le français et le choix de la source de départ pour la construction du corpus de travail}

La question primordiale qui se pose logiquement lors de l'étude des emprunts à l'allemand est celle de savoir quels sont les lexèmes en question et en quelle quantité ceux-ci sont représentés dans le lexique français. C'est ici que surgit le premier problème ; les informations sur le nombre des emprunts lexicaux à l'allemand dans le français sont assez contradictoires : Louis Tardivel repère 500 mots d'origine allemande dans l'Encyclopédie universelle française (1960) mais seulement 167 dans le Grand Larousse de la Langue Française (1975) (TARDIVEL 1991 : 501-502), Henriette et Gérard Walter donnent 652 mots empruntés à l'allemand (de toutes les époques sauf le germanique ancien) (WALTER 1998: 403 ; 270), Paul Fischer (FIscher 1991), qui se concentre sur le lexique du XIXe siècle, tra-

\footnotetext{
Le procédé est en cours d'exécution.
} 
vaille avec 791 emprunts à l'allemand et Walburga Sarcher (Sarcher 2001), qui étudie le lexique du $X X^{\text {e }}$ siècle, en cite même $1500 .^{4}$

Vu le déséquilibre des données dans la littérature spécialisée, j'ai donc choisi de prendre pour point de départ pour mon travail le TLF(i). L'une des raisons est mon effort déjà mentionné de prendre le relais, d'une certaine manière, du programme TLF-Étym, l'autre raison est le fait que le TLF(i) comporte le lexique du XIX ${ }^{\mathrm{e}}$ et $\mathrm{XX}^{\mathrm{e}}$ siècle et qu'il reste toujours un des dictionnaires historiques et étymologiques français les plus riches et les plus fiables, servant de référence à bien d'autres dictionnaires plus récents.

Suite à la recherche avancée dans la version informatisée du TLF, on obtient 277 lexèmes qui sont considérés comme emprunts lexicaux à l'allemand, à l'aide d'une recherche plus détaillée ${ }^{5}$ il est possible d'en isoler une centaine d'autres. L'ensemble de départ pour l'analyse suivante compte donc un peu moins de 400 lexèmes.

\subsection{Délimitation plus précise du corpus de travail et classification des emprunts lexicaux à l'allemand}

Pourtant, la construction du corpus de travail n'est pas achevée avec la sélection des ca 400 lexèmes que le TLF (i) tient pour des emprunts à l'allemand. L'ensemble des lexèmes ainsi désignés se montre assez incohérent et dans de nombreux cas, l'origine allemande de certains emprunts se révèle problématique, voire discutable. Il est donc nécessaire de fixer des critères précis pour la formation du corpus final des lexèmes à analyser, ce qui offre en même temps une possibilité de classifier les emprunts à l'allemand suivant divers angles de vue.

\subsubsection{Aspect étymologique}

\subsubsection{1. Étymons des emprunts ; étymologie directe / étymologie lointaine}

Dans le TLF(i), on rencontre des emprunts dont l'origine est vraiment allemande, p. ex. le mot glass < all. Glas, aussi bien que ceux dont le transfert vers le français s'est opéré par l'intermédiaire de l'allemand mais qui ne sont pas d'origine allemande, p. ex. obus < all. Haubitze < tch. houfnice. Les lexèmes du deuxième type ont été exclus du corpus de travail.

Ensuite, sont à distinguer les emprunts à l'allemand directs et indirects : suite au TLF(i), p. ex. le substantif führer < all. Führer a été adopté directement, tandis que p. ex. le mot halte a été emprunté par l'intermédiaire de l'italien (ital. alto < all. Halt). ${ }^{6}$ Je considère néanmoins que, qu'il s'agisse d'un transfert lexical direct ou d'un emprunt qui s'est opéré par l'intermédiaire d'une autre langue (ou même plusieures), il est question dans tous les cas d'une unité lexicale d'origine allemande et voilà pourquoi j'ai intégré les deux types d'emprunts dans l'ensemble des lexèmes à analyser.

\footnotetext{
4 Il faut noter cependant que la majeure partie du lexique examiné par Fischer et Sarcher appartient au domaine des sciences naturelles et que tous les dérivés des emprunts sont également compris dans cet ensemble.

En consultant ca 3000 notices contenant l'expression « all. » dans un paragraphe quelconque.

6 «De l'ital. alto « arrêt au cours d'une marche (surtout en parlant d'une armée) » (fin XVe-début XVI s. ds l'expr. fare alto « s' arrêter (d'une armée) » ds BATT. t. 1, 1961, lui-même empr. à l'all. Halt terme milit. " arrêt ») impér. subst. de halten « arrêter ». L'h- du fr. est dû à l'all. Halt de même sens. », cf. TLF(i).
} 


\subsubsection{L'emprunt des néologismes allemands formés avec des éléments non-alle- mands}

Un autre groupe de lexèmes considérés comme emprunts à l'allemand par le TLF(i) sont les néologismes allemands adoptés dans le français, qui néanmoins ont été formés à partir de mots d'origine autre qu'allemande. Dans la plupart des cas, il s'agit des composés, p. ex. le mot écologie < all. Ökologie < gr. oikos $+\log _{0}{ }^{7}$, ou de dérivés formés à l'aide d'affixes (p. ex. piétiste < all. Pietist < lat. pietas + suf. -ist). Tous les lexèmes de ce genre ont été exclus, à l'exception de ceux dont au moins une partie formative est allemande, p. ex. plexiglas < lat. plexus + all. Glas.

\subsubsection{Les dérivés des noms propres}

Relativement souvent, on rencontre des emprunts à l'allemand issus d'une formation déonomastique. Pour l'illustrer, on peut citer le dérivé d'un nom de personne - bandonéon < nom de l'Allemand Heinrich Band + suff. -eon (suivant l'all. Orpheon), ou le dérivé d'un toponyme - brandebourg; «Du nom de la province all. de Brandebourg (Brandenburg) dont Berlin est le centre. $»^{8}$ Tous les lexèmes formés à partir d'un nom propre de provenance germanophone ont été inclus dans le corpus de travail.

\subsubsection{4 (In)certitude du TLF(i) dans l'étymologie des unités lexicales examinées}

Les auteurs du TLF(i) ne présentent pas l'étymologie des lexèmes étudiés de manière toujours univoque. La partie «Étymologie et histoire » des notices traitées dans le TLF(i) est souvent introduite par la constatation « d'origine incertaine » ou "d'origine discutée ». Les emprunts de ce type ont été éliminés de l'ensemble de travail et cela même dans les cas où le TLF(i) rajoute, malgré cette incertitude, une hypothèse étymologique possible, comme p. ex. dans le cas du lexème quiche. ${ }^{9}$

Pour les mêmes raisons, j'ai décidé d'exclure tous les lexèmes pour lesquels le TLF(i) hésite entre deux étymons, le plus souvent entre l'étymon allemand et néerlandais, ce qui est p. ex. le cas du mot spirlin. ${ }^{10}$

En revanche, ont été acceptés les lexèmes où la mesure de probabilité de l'analyse du $T L F(i)$ se rapproche de la certitude, c.-à-d. là où l'explication étymologique contient la remarque "probablement », comme p. ex. dans le lexème flingue. ${ }^{11}$

\subsubsection{Aspect diachronique}

Un autre aspect qui joue un rôle lors du tri des emprunts à l'allemand est l'aspect diachronique. Il est important de prendre en compte tous les degrés évolutifs de la langue source, ainsi que différentes branches des langues germaniques, et de délimiter claire-

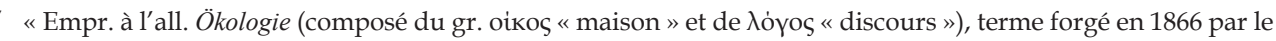
zoologiste et biologiste allemand E. H. Haeckel [1834-1919], relevé dans la préface de son ouvrage Natürliche Schöpfungsgeschichte 1867 (d'apr. Encyclop. brit., s.v. Haeckel); l'empr. à l'all. s'est peut-être fait par l'intermédiaire de l'angl. oecology (1873 tr. Haeckel's Hist. Creat. Pref. ds NED). », cf. TLF(i).

8 Cf. TLF(i).

9 «Mot d'orig. inc., à rapprocher peut-être de l'all. Kuchen « gâteau, tarte ». », cf. TLF(i).

10 «Empr. à l'all. Spierling « éperlan » ou au néerl. spierling « id ». », cf. TLF(i).

11 «prob. empr. au bavarois flinke, flinge « fusil », var. dial. de l'all. Flinte de même sens (cf. FEW t. 15, 2, p. 144b). », cf. $T L F(i)$.
} 
ment lesquelles on va considérer ou pas comme sources légitimes d'emprunts à l'allemand.

Dans mon travail, j'étudie uniquement les lexèmes qui ont été adoptés en français soit à partir de l'allemand qui est parlé sur le territoire de l'Allemagne, l'Autriche ou la Suisse de nos jours (p. ex. gangue < all. Gang ; röstis < all. suisse rösti), ou bien à partir d'un de ses stades historiques précédents, déjà suffisamment différenciés des autres langues germaniques, c.-à-d. l'ancien haut allemand, le moyen haut allemand ou le nouveau haut allemand (p. ex. épeiche < a. h. all. Spëch ; brandevin < m. h. all. Brantwin). J'ai éliminé tous les emprunts que le TLF(i) situe au niveau du germanique, du francique, de l'alémanique etc. (p. ex. robe < germ. occ. *rauba; héberger < a. b. frq. *heribergon; putsch < all. Putsch $<$ além. Putsch).

Ont été naturellement éliminés des emprunts provenant d'autres langues germaniques et de leurs stades évolutifs. Particulièrement déroutants sont les lexèmes empruntés à l'alsacien ; suivant le TLF(i) p. ex. le mot schnick ${ }^{12}$, et par sécurité j'ai également décidé de laisser de côté des lexèmes provenant du (moyen) bas allemand (p. ex. chopine $<\mathrm{m}$. b. all. schope; hisser < b. all. hissen), afin d'éviter des difficultés de différenciation entre le bas allemand et le néerlandais.

\subsubsection{Aspect dialectologique}

À la recherche seront soumises les expressions appartenant à l'un des dialectes allemands ou celles qui ont subi une influence d'un de ces dialectes, comme p. ex. dans le cas du lexème flingue, déjà cité, qui est probablement une variante bavaroise du mot allemand Flinte ${ }^{13}$, ou du lexème rucksack < all. Rucksack, provenant des dialectes alpins, typiques pour le Sud de l'Allemagne. ${ }^{14}$

\subsubsection{Aspect linguistique (morphologie, ortographe, prononciation etc.)}

Le corpus de travail est formé par des unités lexicales qui sont restées sans changement lors du transfert lexical entre l'allemand et le français (p. ex. führer, L.S.D.), aussi bien que par de nombreuses autres qui, après l'adoption dans le français, ont encore évolué ; que ce soit au niveau de la morphologie, de l'orthographe, de la prononciation ou dans un autre domaine - les lexèmes de ce type représentent d'ailleurs la majorité de l'inventaire final. Voyons quelques exemples illustratifs : dans le cas de certains emprunts, on peut noter le changement de genre (p. ex. burg, s. m. < Burg, s. f.), souvent, on observe des changements d'orthographe (glass < Glas) ou même des changements de prononciation (glaçure [glasy:R] < Glasur [glazu:r]). Même des modifications vocaliques et consonantiques plus marquantes ne sont pas rares ; notons p. ex. le mot français nouille par rapport à son étymon allemand Nudel ou le français espiègle, provenant du nom propre allemand Eulenspiegel. Il est à remarquer dans d'autres lexèmes le changement de partie du discours (loustic m., subst./adj. < lustig, adj.). Sur le champ de la morphologie, on peut

\footnotetext{
12 «Empr. au dial. all. de Lorraine (M. F. Follmann, Wörterbuch der deutsch-lothringischen Mundarten, Leipzig, 1909) ou d'Alsace schnick « eau-de-vie ", issu du verbe dial. all. schnicken " effectuer un mouvement rapide avec les mains ou les jambes » (D. Behrens, Beiträge zur fr. Wortgeschichte und Grammatik, Halle, 1910, pp. 4849). ", cf. $T L F(i)$.

${ }_{13}$ Cf. note 12 .

14 «All. Rucksack, de même sens que le fr., mot des dial. alpins, comp. de la forme sans inflexion, propre à l'Allemagne du Sud, de l'all. Rücken « dos » et de Sack «sac » ", cf. TLF(i).
} 
parfois observer une suffixation (griblette < all. Grieben), une apocope $($ stem $(m)$ - apocope de l'all. Stemmbogen) etc.

Il ne faut pas non plus omettre diverses variantes d'une seule unité lexicale ; p. ex. schnouf(f), handball/hand-ball ou backfis(c)h.

\subsubsection{Aspect stylistique}

\subsubsection{Termes scientifiques}

Si l'on veut analyser la mesure de connaissance et d'utilisation d'un certain vocabulaire chez les Français natifs quelconques, il faut logiquement éviter tous les termes spécialisés que l'on pourrait très probablement retrouver uniquement dans des publications spécialisées aux disciplines scientifiques concrètes et parmi les usagers consacrés de cette terminologie, dans des situations communicationnelles adéquates. De mon ensemble d'emprunts à l'allemand ont donc été exclues toutes les notions appartenant aux domaines de la chimie (p. ex. cadmium), de la biochimie (valine), de la médecine (râpes), de la minéralogie (blende), de la géologie (horst), de la psychologie (gestalt), de l'archéologie (vidrecome), de l'historie (hanse), de la musicologie (cromorne), de la littérature (minnesinger), de la numismatique (batz), de la mécanique (came), de la métallurgie (schlich) et d'autres disciplines scientifiques. Suite à cette démarche, le corpus de travail s'est considérablement réduit, vu que la plupart des emprunts à l'allemand sont apparus justement dans le domaine de la science.

Ont été conservées seulement quelques expressions de botanique et de zoologie, concrètement les dénominations de plantes et d'animaux (p. ex. trolle, épeiche, edelweiss) parce que je considère que dans ces domaines, même un locuteur disposant d'un degré d'instruction moyen peut posséder certaines connaissances.

Dans la recherche ont été également inclus quelques lexèmes qui proviennent originairement d'une branche scientifique mais qui à mon avis sont devenus tellement communs qu'il font partie, de nos jours, du lexique général, p. ex. L.S.D. ou leitmotiv.

\subsubsection{Archaïsmes historiques}

Les lexèmes qui sont liés à des événements historiques concrets (p. ex. la guerre de trente ans ou la première et la deuxième guerre mondiale) forment une catégorie spécifique des emprunts à l'allemand (p. ex. gestapo, schupo, oflag), aussi bien que ceux qui, bien que dans d'autres contextes, désignent des objets déjà inexistants, p. ex. des armes historiques, des anciennes monnaies, des parties de vêtements etc. (c.-à-d. archaïsmes historiques proprements dits, p. ex. lansquenet, mark, schilling). Ce genre d'expressions sera probablement seulement repérable dans des textes historiques, dans des publications spécialisées en histoire, éventuellement dans des textes littéraires, et la probabilité que ces mots figurent dans des articles de journaux quotidiens et soient utilisés dans la langue quotidienne par les locuteurs natifs actuels est minimale. Il en résulte que ce type d'emprunt ne peut être lui non plus compris dans la recherche.

Seuls certains lexèmes de ce genre ont été exceptionnellement gardés dans le corpus de travail ; ceux qui ont subi un élargissement de sens : p. ex. le mot Führer est avant tout une désignation alternative d'Adolf Hitler, de nos jours, selon le TLF(i), il porte aussi la 
signification de « dictateur $» .{ }^{15}$ Une situation similaire est à observer chez Méphistophélès, le célèbre personnage du Faust de Goethe, qui peut également être le synonyme d'une personne rusée, « ironique et méchante ». ${ }^{16}$

\section{Le corpus de travail résultant}

Le résultat de cette sélection détaillée est donc l'ensemble suivant de 105 emprunts d'origine allemande dont les plus anciens ont été adoptés dans le lexique français à l'époque de l'ancien haut allemand (c.-à-d. ca 750 ap. J. C.) et dont on peut supposer qu'ils font partie du lexique général français.

\begin{tabular}{|c|c|c|}
\hline 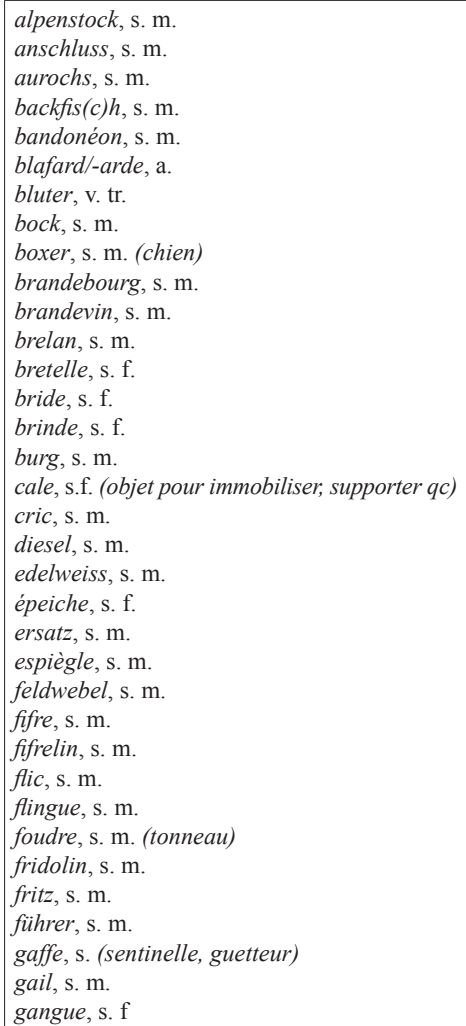 & 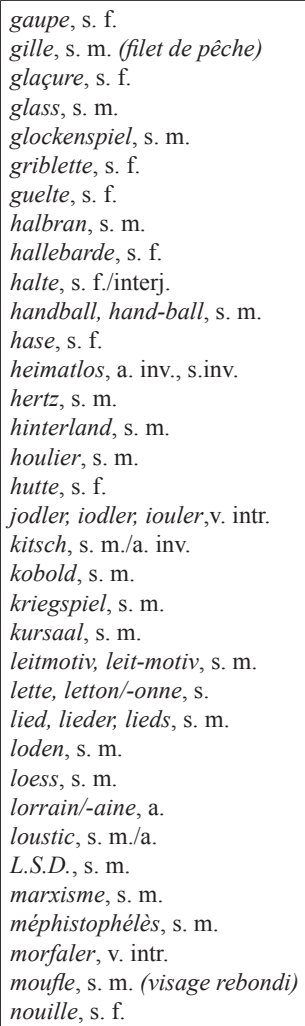 & 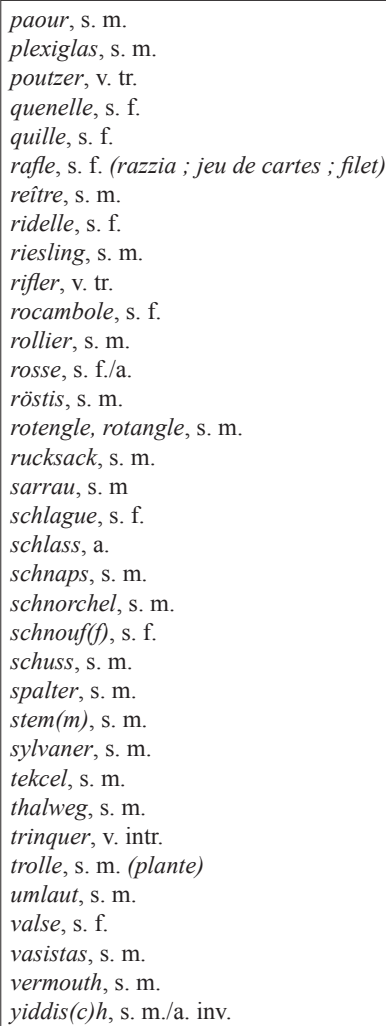 \\
\hline
\end{tabular}

\section{Bibliographie}

FisCHER, Paul (1991), Die deutsch-französischen Beziehungen im 19. Jahrhundert im Spiegel des französischen Wortschatzes, Frankfurt am Main : Verlag Peter Lang (Europäische Hochschulschriften, Reihe XIII, Französische Sprache und Literatur 161).

Imbs, Paul - Quemada, Bernard (dir.) (1971-1994), Trésor de la Langue Française.

15 «1. Titre adopté par Adolf Hitler à partir de 1934. 2. [Précédé d'un art. déf.] Adolf Hitler lorsqu'il portait le titre de « Führer ». [...] B. P. ext. Synon. dictateur (v. Duce, citat. de Guéhenno). », cf. TLF(i).

16 « [P. allus. au personnage du diable dans la légende de Faust] Personne habile, froidement ironique et méchante. », cf. TLF(i). 
Dictionnaire de la langue du XIXe et du XX siècle (1789-1960), 16 volumes, Paris : Éditions du CNRS/Gallimard, http:/ / atilf.atilf.fr/tlf.htm.

SARCHER, Walburga (2001), Das deutsche Lehngut im Französischen als Zeugnis für den Wissenstransfer im 20. Jahrhundert, Hamburg: Verlag Kovač (Schriftenreihe Philologia, Sprachwissenschaftliche Forschungsergebnisse 46).

TARDIVEL, Louis (1991), Répertoire des emprunts du français aux langues étrangères, Sillery : Septentrion.

WALter, Gérard - Walter, Henriette (1998), Dictionnaire des mots d'origine étrangère, Paris : Larousse.

\author{
Zuzana Navrátilová \\ Katedra romanistiky \\ Filozofická fakulta \\ Univerzita Palackého v Olomouci \\ Křížkovského 10 \\ 77180 Olomouc \\ République tchèque \\ zuzka.navr@volny.cz
}

